\title{
Caracterización citogenética del pez neotropical Brycon henni (Pisces: Characidae)
}

\author{
Diana David López ${ }^{1}$, Gonzalo Vásquez Palacio ${ }^{2}$, Tatiana Ruiz Cortés ${ }^{1} \&$ Marta Olivera Angel $^{1}$ \\ 1. Grupo Biogénesis, Universidad de Antioquia, Carrera 75 No 65-87, AA 1226, Medellín, Colombia. Telefax: (574) \\ 4259149; dianahumus@gmail.com; ztatiana@yahoo.com; syngamia@gmail.com \\ 2. Unidad de Genética Médica, Facultad de Medicina, Universidad de Antioquia, Carrera 51 D No. 62 - 29, AA 1226, \\ Medellín, Colombia. Tel: (574) 2106063; gvasquezp@gmail.com
}

Recibido 14-VIII-2007. Corregido 30-VI-2008. Aceptado 31-VII-2008.

\begin{abstract}
Cytogenetic characterization of the neotropical fish Brycon henni (Pisces: Characidae). Brycon henni, is a native fish from Western Colombia is important as food and in sport fishing, and could be cultured in waters between 18 and $28^{\circ} \mathrm{C}$. A previous cytogenetic study in branchial cells indicated different chromosomal complements. Total blood samples were seeded in $4 \mathrm{ml}$ Ham-F12 culture medium, supplemented with $0.5 \mathrm{ml}$ Bovine Fetal Serum and $0.7 \mathrm{ml}$ Phyitohemaglutinin $\mathrm{M}$ (Gibco $\left.{ }^{\circledR}\right)$ during $96 \mathrm{hr}$ at $28^{\circ} \mathrm{C}$; an antimitotic factor $(100 \mu$ l Colcemid $(10 \%)$ was added. Cells were incubated in $10 \mathrm{ml} \mathrm{KCl}$ hypotonic solution $(0.56 \%)$ for 24 min at $37^{\circ} \mathrm{C}$, fixed in methanol; acetic acid $(3: 1)$ three times, separated by centrifugations, extended drip, and the chromosomes slides were stained with $5 \%$ Giemsa (5\%). The best metaphases (6 males and 8 females) were photographed. There was a diploid number of 50 chromosomes: Metacentrics (26M), Submetacentrics (16SM) and Subtelocentrics (8ST). The fundamental number is $100 \mathrm{arms}$. The evolutionary tendency is type B with no supernumerary chromosomes; a first pair of big metacentric chromosomes is characteristic for Brycon, while no chromosomic sexual heteromorfism was detected. Rev. Biol. Trop. 56 (4): 1619-1628. Epub 2008 December 12.
\end{abstract}

Key words: Karyotype, chromosomes, fish, bryconids, lymphocytes.

La sabaleta Brycon henni (Eigenmann 1913), es un pez nativo de agua dulce, que se encuentra ampliamente distribuido en la región occidental de Colombia (Montoya-López et al. 2006). Su importancia radica en la seguridad alimentaria que brinda a las poblaciones ribereñas, a sus características combativas para la pesca deportiva y a que es una especie promisoria de cultivo para las zonas de clima templado, es decir, entre 18 y $28^{\circ} \mathrm{C}$ (Perdomo 1978). Estudios relacionados con la morfología (Miles 1947, Dahl 1971), los hábitos alimenticios (Builes y Urán 1974), la reproducción (Builes y Urán 1974, Molina 1982, Montoya- López et. al. 2006), el mantenimiento en cautiverio (Perdomo 1978), la diversidad genética (Pareja 2002, Pineda Santis 2007) y algunos aspectos citogenéticos (De Greiff y Montoya 1988). Sin embargo, estudios de citogenética a fondo y con procedimientos alternativos como el cultivo de linfocitos, no han sido realizados.

La citogenética en los peces ha servido para la clasificación taxonómica (Sota 1982, Uribe- Alcocer et al. 1983, Durán- González et al. 1990), para el análisis de la tendencia evolutiva, filogenia y rearreglos cromosómicos (Uyeno y Miller 1973, Thompson 1979, Salas y Boza 1991, Vicente et al. 1996, Nakayama et al. 2002), para estudios de biodiversidad (Fenocchio et al. 2000), para la caracterización de polimorfismo sexual (Bertollo et al. 2000, Sola et al. 1990), para la determinación de hibridaciones para mejoramiento de la producción y la viabilidad de genotipos (Howell y 
Villa 1976, Salas y Boza 1991, Burbano 2001, Boron 2003, Porto-Foresti et al. 2004) y para la observación de polimorfismos y especiaciones (Chen y Ebeling 1971, Sola et al. 1990, Bertollo et al. 2000, Nakayama et al. 2001).

Del género Brycon en Colombia, es conocido el cariotipo de B. amazonicus (Parada et al. 2003) y en 1988, De Greiff y Montoya realizaron estudios para B. fowleri, B. medemi, B. moorei y $B$. henni con células branquiales. En $B$. henni encontraron diferentes complementos cromosómicos: 46, 47, 48, 49 y 50, el de mayor frecuencia de aparición fue 48 (50.3\% de las muestras) y sugirieron que el primer par cromosómico eran los pares sexuales; la morfología cromosómica con mayor aparición fue acrocéntrica y subtelocéntrica, encontrándose también cromosomas supernumerarios. Concluyeron que la variabilidad de resultados pudo deberse a los polimorfismos por procesos de especiación y a la retracción de los cromosomas en los preparados.

El objetivo de este trabajo es el de caracterizar el cariotipo de $B$. henni por medio de la técnica de cultivo de linfocitos lo que evita el sacrificio de los animales y comparar los resultados con aquellos informados por la literatura.

\section{MATERIALES Y MÉTODOS}

Material biológico: Muestras de sangre de veintiséis especímenes adultos, nueve correspondían a machos y diecisiete a hembras que son mantenidos en condiciones de cautiverio en el Centro Experimental y de Producción Piscícola del Politécnico Jaime Isaza Cadavid, Municipio de San Jerónimo, Antioquia, Colombia (06 $26^{\circ} 49^{\prime}$ ' N, 75 43' $\left.58^{\prime} \mathrm{O}\right)$. Se identificaron por sus características morfológicas, siguiendo la taxonomía descrita por Dahl (1971). Especímenes testigos de Brycon henni se encuentran en el Instituto Alexander von Humboldt, Claustro de San Agustín, Villa de Leyva, Boyacá, Colombia (Referencia: IavHP4304 y IavHP 4355).

Toma de muestras: Las muestras de sangre se tomaron por punción de la vena dorsal con una jeringa de $2 \mathrm{ml}$ estéril heparinizada (D. David, en prep.) identificando el sexo por observación de la papila urogenital, de la aleta anal y por presión abdominal para extracción de gametos. Las muestras se transportaron en el émbolo de la misma jeringa, marcada y envuelta con papel aluminio, a $4^{\circ} \mathrm{C}$ hasta la Facultad de Ciencias Agrarias de la Universidad de Antioquia en la ciudad de Medellín (6 $6^{\circ} 14$ ' 9.33" N, $75^{\circ} 34^{\prime} 30.49$ ” O) para su procesamiento.

Obtención de cromosomas y cariotipo: El cultivo se realizó sembrando $0.5 \mathrm{ml}$ de sangre periférica en $4 \mathrm{ml}$ de medio HAM F12 (Sigma®) suplementado con $0.5 \mathrm{ml} \mathrm{de}$ Suero Fetal Bovino SFB y $0.7 \mathrm{ml}$ de mitógeno Fitohemaglutinina M PHA- M (Gibco®) al $0.5 \%$ e incubando a $28^{\circ} \mathrm{C}$ durante $96 \mathrm{hrs}$ y 1.5 hrs antes de la cosecha se agregaron $100 \mu \mathrm{de}$ Colcemid (Invitrogen ${ }^{\circledR}$ ) al 10\%. Después de la incubación, se realizó la hipotonización adicionando $10 \mathrm{ml}$ de $\mathrm{KCl} 0.56 \%$ a $37^{\circ} \mathrm{C}$ por $24 \mathrm{~min}$ seguido de tres fijaciones sucesivas con metanol: ácido acético (3:1); se separó el contenido celular por centrifugaciones a 500 gravedades por $5 \mathrm{~min}$. El extendido o fijación celular se hizo por goteo en portaobjetos prefijados, fríos $\left(4^{\circ} \mathrm{C}\right)$ y húmedo los cuales después de secados al ambiente se tiñeron con Giemsa al 5\% en buffer de sal fosfatada PBS pH 6.8. Se realizaron en total 15 cultivos con 5 repeticiones cada uno. Cada cultivo con su sus repeticiones correspondían al misma muestra de sangre (al mismo individuo) debidamente identificada.

Para la clasificación cromosómica, los extendidos celulares se observaron en microscopio Zeiss $\mathrm{ACRO}^{\circledR}$ en objetivo $100 \mathrm{X}$ y las metafases fueron fotografiadas. De 500 metafases, fueron seleccionadas las 14 mejores de acuerdo a la claridad y extendido de cada cromosoma que se observara; 6 correspondían a machos y 8 a hembras debidamente identificados; fueron impresas en resolución óptima para identificar el centrómero y a partir de éste se midieron los brazos cromosómicos sobre la impresión obteniendo las medidas en número de puntos (Bertollo et al. 1978) que sólo indica 
morfología y no tamaño real ya que no se usó la regla micrométrica.

Los cromosomas se clasificaron de acuerdo a Levan et al. (1964), en donde una relación entre brazo corto (p) y brazo largo (q) de 1.0 a 1.7 es Metacéntrico (M), entre 1.71 y 3.0 es Submetacéntrico (SM), entre 3.1 y 7.0 Subtelocéntrico (ST) y superior a 7.0 es Acrocéntrico (A). Los datos mencionados anteriormente se les calcularon la media y desviaciones estándar. Se determinó el Número Fundamental NF que es el total de brazos cromosómicos.

\section{RESULTADOS}

El análisis de las metafases mostró un número diploide de $2 \mathrm{n}=50$ cromosomas para el total de ellas. El número fundamental fue igual a cien $(\mathrm{NF}=100)$ y la clasificación cromosómica de 26 M, 16 SM y 8 ST (Fig. 1 y Cuadro 1 ). El primer par de cromosomas metacéntricos presentan un tamaño superior comparado con el resto del complemento (Fig. 2). No se observan diferencias morfológicas entre las metafases de machos y hembras, por lo tanto, no se determinó la existencia de dimorfismo sexual cromosómico.

\section{DISCUSIÓN}

El cariotipo de $B$. henni mostró como número diploide $2 \mathrm{n}=50$ cromosomas birrámeos, que fueron clasificados en 26M, $16 \mathrm{SM}$ y 8ST (Fig. 1 y Cuadro 1). Debido a que todos los cromosomas mostrados por el cariotipo de la sabaleta fueron birrámeos, el número fundamental fue de 100 brazos cromosómicos, igual a los demás del género Brycon. Estos resultados difieren del estudio citogenético realizado previamente en la especie de que trata el estudio, en donde se identificaron $2 \mathrm{n}=48$ cromosomas que fueron clasificados como M, SM, ST y A y presencia de cromosomas supernumerarios (Greiff y Montoya 1988). Sin embargo, las investigaciones más recientes indican que el número de cromosomas común para el género Brycon es de $2 \mathrm{n}=50$, con morfología M, SM y ST y proporción de brazos cromosómicos de 100 (Wasko y Galetti 2000), sin la observación de cromosomas supernumerarios. Las diferencias entre estas especies son en la

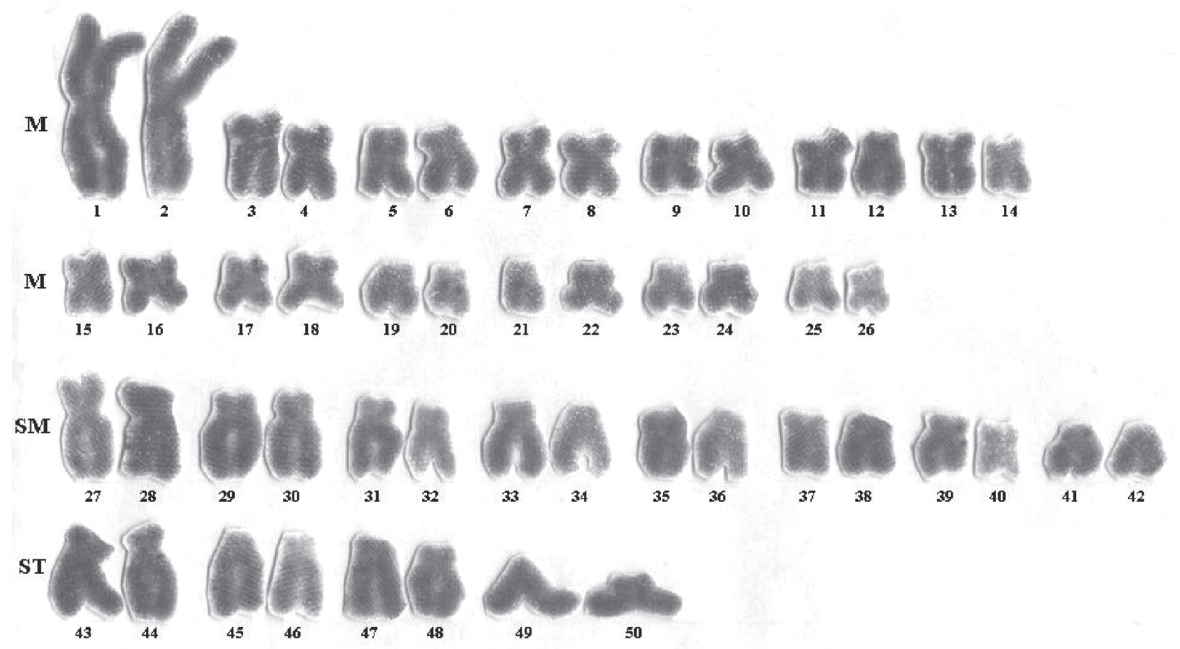

Fig. 1. Complemento cromosómico de B. henni. Presenta 50 cromosomas clasificados en 26 Metacéntricos (M), 16 Submetacéntricos (SM) y 8 Subtelocéntricos (ST). Giemsa $5 \%$.

Fig. 1. Chromosomic complement of the fish B. henni. It has 50 chromosomes: 26 Metacentric (M), 16 Submetacentric (SM) and 8 Subtelocentric (ST). Giemsa 5\%. 


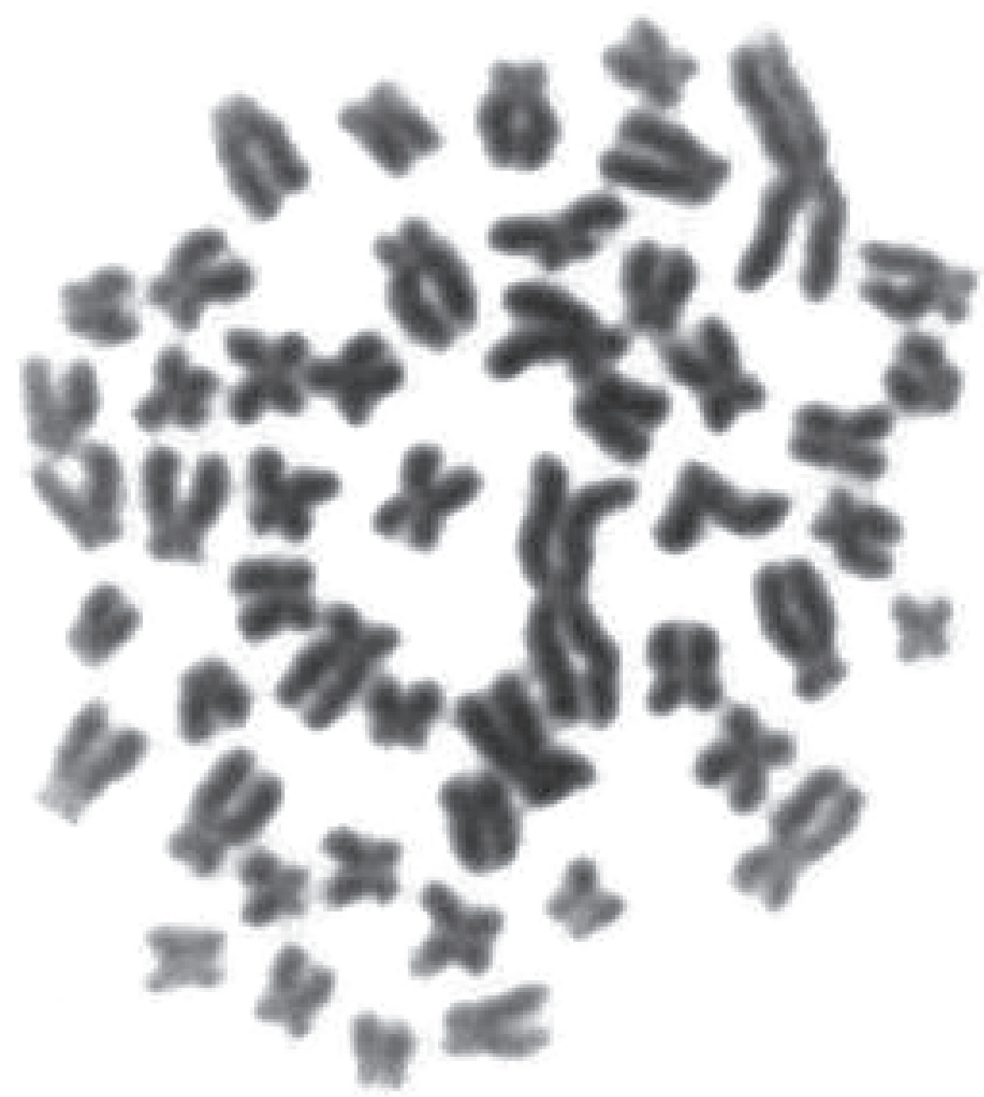

Fig. 2. Metafase de B. henni. Presenta un par cromosómico de gran tamaño, característico del género Brycon. Giemsa 5\%.

Fig. 2. B. henni metaphase. The large pair of chromosomes is characteristic of Brycon. Giemsa 5\%.

distribución de número de cromosomas de acuerdo a su morfología, así: Para $B$. lundii se propuso: $22 \mathrm{M}, 24 \mathrm{SM}, 4 \mathrm{ST}$; para $B$. microlepis: 20M, 24SM, 6ST (Margarido y Galetti 1999); para B. Cf.reinhadti: 22M, 28SM/ST; y para $B$. amazonicus (Parada et al. 2003)

El primer par de cromosomas metacéntricos han mostrado tamaño superior al del resto de los cromosomas del cariotipo; dicha característica ha sido común para las especies del género Brycon por lo que se ha considerado como marcador cromosómico en los bricónidos (Parada et al. 2003). También, De Greiff y Montoya (1988) los consideraron tentativamente como cromosomas sexuales, lo que no fue comprobado en las demás especies de carácidos ni en el presente trabajo. Morfológicamente (por tinción giemsa), estos cromosomas no aparentan diferencias entre sexos. En diferentes especies de la familia Characidae a la que pertenece el Género Brycon, se ha observado este par grande de cromosomas metacéntricos, como en Astyanax scabripinnis (Dos Santos y Morelli 2000), en Serrasalmus rhombeus (Nakayama et al. 2001), Oligosarcus hepsetus (Kavalco et al. 2005) y en Prochilodus reticulatus (Silva 2001).

En $B$. henni se observó que el número diploide es constante tanto en el mismo individuo como en la especie, ya que ninguna de las metafases contabilizadas mostró número diploide diferente a 50 cromosomas lo que es 
CUADRO 1

Cromosomas de 14 metafases de $\mathrm{B}$. henni

TABLE 1

Chromosome from 14 metaphases of $\mathrm{B}$. henni

\begin{tabular}{|c|c|c|c|}
\hline Cromosoma & $\begin{array}{l}\text { Brazo corto }(\mathrm{p})^{*} \\
\pm \text { D.E. }\end{array}$ & $\begin{array}{c}\text { Brazo largo }(\mathrm{q})^{*} \\
\quad \pm \text { D.E. }\end{array}$ & $\begin{array}{l}\text { Longitud Total } \\
\mathrm{p}+\mathrm{q} * \pm \text { D.E. }\end{array}$ \\
\hline 1 & $9.87 \pm 0.30$ & $9.9 \pm 0.33$ & $19.86 \pm 0.53$ \\
\hline 2 & $9.58 \pm 0.80$ & $9.66 \pm 0.69$ & $19.25 \pm 1.48$ \\
\hline 3 & $4.23 \pm 0.82$ & $5.83 \pm 1.02$ & $10.06 \pm 1.72$ \\
\hline 4 & $3.94 \pm 0.72$ & $5.40 \pm 1.07$ & $9.34 \pm 1.66$ \\
\hline 5 & $3.71 \pm 0.67$ & $4.71 \pm 0.82$ & $8.42 \pm 1.34$ \\
\hline 6 & $3.41 \pm 0.59$ & $4.58 \pm 0.81$ & $7.98 \pm 1.28$ \\
\hline 7 & $3.37 \pm 0.68$ & $4.11 \pm 0.75$ & $7.49 \pm 1.26$ \\
\hline 8 & $3.22 \pm 0.54$ & $4.05 \pm 0.79$ & $7.27 \pm 1.18$ \\
\hline 9 & $3.19 \pm 0.66$ & $3.89 \pm 0.57$ & $7.08 \pm 1.12$ \\
\hline 10 & $3.02 \pm 0.54$ & $3.83 \pm 0.65$ & $6.85 \pm 1.03$ \\
\hline 11 & $2.89 \pm 0.46$ & $3.78 \pm 0.64$ & $6.68 \pm 0.97$ \\
\hline 12 & $2.94 \pm 0.45$ & $3.67 \pm 0.65$ & $6.60 \pm 0.97$ \\
\hline 13 & $2.74 \pm 0.52$ & $3.68 \pm 0.51$ & $6.42 \pm 0.93$ \\
\hline 14 & $2.69 \pm 0.36$ & $3.59 \pm 0.67$ & $6.29 \pm 0.96$ \\
\hline 15 & $2.76 \pm 0.49$ & $3.43 \pm 0.59$ & $6.19 \pm 0.95$ \\
\hline 16 & $2.77 \pm 0.48$ & $3.19 \pm 0.52$ & $5.96 \pm 0.89$ \\
\hline 17 & $2.71 \pm 0.49$ & $3.19 \pm 0.53$ & $5.90 \pm 0.88$ \\
\hline 18 & $2.67 \pm 0.58$ & $3.16 \pm 0.47$ & $5.83 \pm 0.95$ \\
\hline 19 & $2.53 \pm 0.64$ & $3.18 \pm 0.53$ & $5.71 \pm 0.99$ \\
\hline 20 & $2.48 \pm 0.43$ & $3.16 \pm 0.63$ & $5.65 \pm 0.91$ \\
\hline 21 & $2.33 \pm 0.58$ & $3.14 \pm 0.57$ & $5.47 \pm 1.03$ \\
\hline 22 & $2.34 \pm 0.48$ & $2.92 \pm 0.79$ & $5.27 \pm 1.22$ \\
\hline 23 & $2.43 \pm 0.67$ & $3.35 \pm 1.44$ & $5.78 \pm 2.05$ \\
\hline 24 & $2.43 \pm 0.74$ & $3.27 \pm 1.41$ & $5.70 \pm 2.07$ \\
\hline 25 & $2.28 \pm 0.60$ & $3.40 \pm 1.56$ & $5.68 \pm 2.08$ \\
\hline 26 & $2.33 \pm 0.69$ & $3.15 \pm 1.55$ & $5.48 \pm 2.19$ \\
\hline 27 & $2.69 \pm 0.89$ & $4.91 \pm 2.10$ & $7.60 \pm 2.90$ \\
\hline 28 & $2.81 \pm 0.64$ & $5.16 \pm 1.84$ & $7.46 \pm 2.80$ \\
\hline 29 & $2.52 \pm 0.38$ & $5.84 \pm 1.41$ & $8.36 \pm 1.73$ \\
\hline 30 & $2.69 \pm 0.62$ & $5.73 \pm 0.68$ & $8.42 \pm 1.23$ \\
\hline 31 & $2.53 \pm 0.47$ & $5.33 \pm 0.68$ & $7.85 \pm 1.00$ \\
\hline 32 & $2.41 \pm 0.45$ & $5.17 \pm 0.64$ & $7.58 \pm 0.98$ \\
\hline 33 & $2.23 \pm 0.41$ & $5.09 \pm 0.62$ & $7.32 \pm 0.87$ \\
\hline 34 & $2.15 \pm 0.41$ & $4.68 \pm 0.62$ & $6.83 \pm 0.92$ \\
\hline 35 & $2.17 \pm 0.28$ & $4.38 \pm 0.63$ & $6.55 \pm 0.82$ \\
\hline 36 & $2.11 \pm 0.33$ & $4.36 \pm 0.62$ & $6.46 \pm 0.84$ \\
\hline 37 & $1.96 \pm 0.43$ & $4.22 \pm 0.60$ & $6.18 \pm 0.96$ \\
\hline 38 & $1.97 \pm 0.40$ & $3.99 \pm 0.58$ & $5.96 \pm 0.93$ \\
\hline 39 & $1.91 \pm 0.37$ & $4.16 \pm 1.19$ & $6.07 \pm 1.42$ \\
\hline 40 & $1.78 \pm 0.40$ & $4.02 \pm 0.94$ & $5.80 \pm 1.26$ \\
\hline 41 & $1.81 \pm 0.36$ & $4.08 \pm 1.40$ & $5.89 \pm 1.64$ \\
\hline 42 & $1.79 \pm 0.32$ & $4.62 \pm 1.70$ & $6.41 \pm 1.84$ \\
\hline 43 & $1.89 \pm 0.36$ & $6.43 \pm 2.10$ & $8.33 \pm 2.31$ \\
\hline 44 & $1.84 \pm 0.35$ & $6.15 \pm 1.44$ & $7.99 \pm 1.71$ \\
\hline 45 & $1.69 \pm 0.30$ & $6.11 \pm 0.93$ & $7.80 \pm 1.04$ \\
\hline 46 & $1.56 \pm 0.33$ & $6.12 \pm 0.78$ & $7.68 \pm 0.99$ \\
\hline 47 & $1.64 \pm 0.28$ & $5.82 \pm 0.81$ & $7.46 \pm 1.02$ \\
\hline 48 & $1.43 \pm 0.35$ & $5.31 \pm 0.98$ & $6.74 \pm 1.24$ \\
\hline 49 & $1.27 \pm 0.32$ & $4.68 \pm 0.90$ & $5.95 \pm 1.09$ \\
\hline 50 & $1.17 \pm 0.37$ & $4.23 \pm 1.00$ & $5.41 \pm 1.32$ \\
\hline
\end{tabular}

I. C. $(\mathrm{p} / \mathrm{p}+\mathrm{q}) 100$ \pm D.E.

$49.69 \pm 0.85$

$49.76 \pm 0.62$

$42.03 \pm 3.18$

$42.34 \pm 3.46$

$44.07 \pm 3.98$

$42.72 \pm 3.53$

$44.97 \pm 4.40$

$44.36 \pm 4.14$

$44.93 \pm 3.85$

$44.12 \pm 4.06$

$43.41 \pm 3.93$

$44.67 \pm 4.02$

$42.49 \pm 3.69$

$43.12 \pm 3.66$

$44.63 \pm 4.37$

$45.91 \pm 4.13$

$45.59 \pm 4.13$

$43.96 \pm 5.36$

$44.14 \pm 4.53$

$42.46 \pm 4.63$

$44.85 \pm 3.95$

$42.74 \pm 5.01$

$43.18 \pm 4.79$

$41.28 \pm 5.06$

$43.81 \pm 5.02$

$36.88 \pm 5.86$

$36.99 \pm 6.86$

$30.95 \pm 5.02$

$31.67 \pm 3.19$

$32.11 \pm 3.54$

$31.76 \pm 3.37$

$30.46 \pm 3.63$

$31.43 \pm 3.27$

$33.22 \pm 2.82$

$32.62 \pm 3.17$

$31.56 \pm 3.34$

$32.92 \pm 2.85$

$31.78 \pm 3.89$

$30.76 \pm 3.27$

$31.51 \pm 4.34$

$29.24 \pm 6.22$

$23.74 \pm 5.73$

$23.43 \pm 3.66$

$21.81 \pm 4.13$

$20.23 \pm 2.97$

$21.92 \pm 2.16$

$21.06 \pm 3.22$

$21.29 \pm 3.81$

$21.46 \pm 3.02$
$46.37 \pm 3.89$
R. B. $q / p$

\pm D.E.

$1.01 \pm 0.04$

$1.01 \pm 0.03$

$1.39 \pm 0.18$

$1.38 \pm 0.18$

$1.29 \pm 0.21$

$1.36 \pm 0.18$

$1.24 \pm 0.22$

$1.27 \pm 0.21$

$1.24 \pm 0.20$

$1.28 \pm 0.21$

$1.32 \pm 0.21$

$1.37 \pm 0.20$

$1.33 \pm 0.19$

$1.26 \pm 0.23$

$1.17 \pm 0.19$

$1.20 \pm 0.21$

$1.21 \pm 0.21$

$1.31 \pm 0.28$

$1.29 \pm 0.24$

$1.38 \pm 0.24$

$1.25 \pm 0.20$

$1.37 \pm 0.27$

$1.34 \pm 0.26$

$1.46 \pm 0.36$

$1.31 \pm 0.28$

$1.78 \pm 0.48$

$1.78 \pm 0.45$

$2.30 \pm 0.43$

$2.19 \pm 0.33$

$2.15 \pm 0.35$

$2.18 \pm 0.33$

$2.33 \pm 0.39$

$2.21 \pm 0.34$

$2.03 \pm 0.27$

$2.09 \pm 0.31$

$2.21 \pm 0.34$

$2.06 \pm 0.27$

$2.20 \pm 0.48$

$2.29 \pm 0.37$

$2.24 \pm 0.55$

$2.60 \pm 0.92$

$3.43 \pm 1.01$

$3.35 \pm 0.59$

$3.72 \pm 0.80$

$4.05 \pm 0.82$

$3.61 \pm 0.49$

$3.88 \pm 0.95$

$3.87 \pm 1.05$

$3.75 \pm 0.69$
$1.26 \pm 0.20$

Clasificación

M

$\mathrm{M}$

$$
\text { M }
$$$$
\text { M }
$$$$
\text { M }
$$$$
\text { M }
$$$$
\text { M }
$$$$
\text { M }
$$$$
\text { M }
$$$$
\begin{aligned}
& \text { M } \\
& \text { M }
\end{aligned}
$$$$
\text { M }
$$$$
\text { M }
$$$$
\begin{aligned}
& \mathrm{M} \\
& \mathrm{M}
\end{aligned}
$$$$
\begin{aligned}
& \mathrm{M} \\
& \mathrm{M}
\end{aligned}
$$$$
\text { M }
$$$$
\text { M }
$$$$
\text { M }
$$$$
\text { M }
$$$$
\mathrm{M}
$$$$
\text { M }
$$$$
\text { M }
$$$$
\text { M }
$$$$
\text { M }
$$$$
\begin{gathered}
\text { M } \\
\text { SM }
\end{gathered}
$$$$
\text { SM }
$$$$
\text { SM }
$$$$
\text { SM }
$$$$
\text { SM }
$$$$
\text { SM }
$$$$
\text { SM }
$$$$
\text { SM }
$$$$
\begin{aligned}
& \text { SM } \\
& \text { SM }
\end{aligned}
$$$$
\text { SM }
$$$$
\text { SM }
$$$$
\text { SM }
$$$$
\text { SM }
$$$$
\text { SM }
$$$$
\text { SM }
$$$$
\text { SM }
$$$$
\text { ST }
$$$$
\text { ST }
$$$$
\text { ST }
$$$$
\text { ST }
$$$$
\text { ST }
$$$$
\text { ST }
$$$$
\text { ST }
$$$$
\text { ST }
$$

* En número de puntos

D. E. = Desviación estándar

I. C. = Indice Centromérico

R. B. = Relación de Brazo
$2 \mathrm{n}=50$

$\mathrm{n}=25$

Número Fundamental $(\mathrm{NF})=100$ 
contrario a lo hallado por De Greiif y Montoya en 1988. De acuerdo a otros autores (Margarido y Galetti 1999, Parada et al. 2003 y Brasessco 2004), se podría considerar que las especies del género Brycon presentan un ancestro común con un número cromosómico básico y cariotipos simétricos, en donde los rearreglos cromosómicos no son comunes, lo que no produciría alteraciones del cariotipo en el tiempo (Brasessco 2004).

No sólo el género Brycon conserva estas similitudes entre sus especies, también otros peces pertenecientes al mismo orden (Characiforme) como el género Bryconamericus mantienen un número diploide de 52 cromosomas (Paintner-Marques et al. 2003); cinco especies del género Serrasalmus con 60 cromosomas considerándole como el número diploide ancestral del género (Nakayama et al. 2002) y las familias Curimatidae, Prochilodontidae, Anostomidae y Parodontidae con $2 \mathrm{n}=54$ cromosomas M y SM (Brasessco 2004), dato confirmado en las especies estudiadas del género Prochilodus que son de la familia Curimatidae (Silva 2001). Igualmente, estos hallazgos han sido publicados en especies de otros órdenes como el Siluriforme, con cinco del género Trichomycterus que tienen 54 cromosomas (Sato et al. 2004) y los de la familia Pimelodidae Sorubim cuspicaudus, Pseudoplatystoma fasciatum y $P$ tigrinum de Colombia y Steindachneridion scripta de Brasil con 2 n=56 cromosomas (Camacho- Garzón y Burbano 1999a, Valenzuela 2001 y Swarça et al. 2005). Esta información llevaría a pensar que las diferencias entre las especies ícticas son dadas por la cantidad de cromosomas de determinada morfología y sus rearreglos micro-estructurales, lo que en el momento de especiación sería más importante que el número diploide. Por ejemplo, al analizar los cariotipos estructuralmente conservados de la familia Curimatidae con Bandas C y NOR, los autores afirmaron que diferencias entre sus especies estaban acompañadas de rearreglos micro-estructurales, evidenciado por la diversa distribución de la heterocromatina y por las bandas NOR (Brassesco et al. 2004).
Aunque de manera artificial se podrían obtener híbridos entre bricónidos por la similitud de los cariotipos, siendo algunos simpátri$\cos$ (cariotipos sin formas intermedias) (Born y Bertollo 2001) como los de B. cephalus y los de B. Amazonicus, no es esto posible de forma natural ya que se encuentran aislados reproductivamente por barreras físicas, ambientales y ecológicas por la distribución en diversos ecosistemas de América Central y del Sur (Dahl 1971) que han impedido el flujo genético entre poblaciones. Así, la similitud y estabilidad del cariotipo entre los bricónidos indica que los rearreglos cromosómicos no son los únicos requisitos para la especiación, como lo afirmaron Howell y Villa (1976) que al cruzar Rhnichthys atratulurs y $R$. cataractae con cariotipos idénticos obtuvieron descendientes estériles. Se ha observado que el número diploide de los híbridos se conserva con respecto al de los parentales. Por ejemplo, los híbridos de Piaractus brachypomus y Colossoma macropomum y sus retrocruces F1 x parentales de las dos primeras especies, tienen un número diploide de 54 cromosomas (Martino 2004); los híbridos de las carpas Aristichthys nobilis y Hypophthalmichthys molitrix presentaron $2 \mathrm{n}=48$ cromosomas pero diferente morfología permitiendo distinguirlos de los parentales (De Almeida- Toledo et al.1995); esto último no sucedió al analizar los cariotipos de Oreochromis urolepis hornorum y $O$. mossambicus y sus híbridos, donde la morfología cromosómica fue igual, sugiriendo un tipo de especiación diferente a la diversificación cariotípica mencionada anteriormente (Arreguín- Espinosa y Uribe-Alcocer 1989).

Thompson (1979) mencionó dos tipos de tendencias evolutivas de los peces de acuerdo a la morfología cromosómica: los de tendencia Subtelocéntrico-Acrocéntrico (stt) que son menos evolucionados, denominada Tipo A, siendo el cariotipo ancestral de los peces, es decir la especie más primitiva, de 48 cromosomas stt ya que es el número diploide más presentado en el caso de algunos cíclidos neotropicales (Thompson 1979) y más del 51\% en los cíclidos africanos (Klinkhardt et al. 1995); y los 
de tendencia Meta- Submetacéntricos (msm) que son más evolucionados denominada Tipo $\mathrm{B}$, así tengan más o menos de 48 cromosomas. Antes de Thompson (1979) ya se hablaba de la tendencia evolutiva de los peces hacia cromosomas msm como fue la comparación entre Gillichthys mirabilis y G. seta en donde Chen y Ebeling 1971 dicen que el segundo es más especializado por tener cromosomas $\mathrm{M}$ y menos $\mathrm{A}$, mientras que el primero no tiene $\mathrm{M}$ y presenta mayormente A. Esto se explica por un posible rearreglo intracromosomal por medio de inversión pericéntrica que transformó los cromosomas Acrocéntricos a Metacéntricos y Subtelocéntricos en G. seta. En peces perciformes han mostrado un mecanismo de reducción de cromosomas por fusión céntrica en donde las especies más primitivas (Seriola nigrofasciata y varios del género Chromis) tenían $2 n=48$ cromosomas Acrocéntricos, y los más evolucionados (por ejemplo Badis badis y $C$. flavixuada) presentaron reducción de cromosomas con presencia de metacéntricos (Tripathy y Das 1988 y Molina y Galetti 2002). Igualmente, dentro de los cíclidos cuyo número diploide es 48 cromosomas, la tribu Tilapiine se redujo a 44 cromosomas, aplicándose la hipótesis evolutiva de las especies anteriores (Martins et al. 2004). Dentro de los loricáridos, la evolución del cariotipo se ha dado por fusiones y fisiones cromosómicas, en donde las especies de menor número diploide presentan cromosomas birrámeos (dos brazos) y los de mayor número diploide no los presentan, indicando diferentes vías evolutivas dentro de sus subfamilias obteniendo gran diversidad de cariotipos. La subfamilia Upsilodinae es considerada como la más basal y su especie Upsilodus sp con la de mayor número cromosómico dentro de los loricáridos, con 96 cromosomas en su mayoría acrocéntricos (Kavalco et al. 2005).

De acuerdo a lo anterior, para B. henni se puede decir que tanto ella como su género, especies del neotrópico, son más evolucionadas ya que presentan más cromosomas M - SM que ST y no de A, es decir que de acuerdo a Thompson (1979), la tendencia evolutiva de $B$. henni sería de Tipo B.
Se hace necesario complementar la información con respecto a la variación genética en las poblaciones de B. henni ((Pareja, 2000 y Pineda Santis et al. 2007). dándole continuidad al estudio citogenético con la aplicación de las técnicas necesarias para determinar variaciones asociadas al ambiente, marcadores (Saito et al. 2007) y analizar la existencia del heteromorfismo sexual (Phillips et. al. 2001), polimorfismos y demás estudios acordes a la citogenética para la toma de decisiones en términos biológicos, reproductivos y productivos como lo han hecho con otros géneros dentro la clase peces.

\section{AGRADECIMIENTOS}

El presente estudio tuvo financiamiento parcial de la Universidad de Antioquia-CODI, mediante el fondo para proyectos de investigación para estudiantes de pregrado-2004. Los autores agradecen las facilidades proporcionadas por Centro Experimental y de Producción Piscícola del Politécnico Jaime Isaza Cadavid del Departamento de Antioquia, Colombia.

\section{RESUMEN}

La sabaleta Brycon henni, es un pez nativo del occidente colombiano importante para la seguridad alimentaria de las poblaciones ribereñas y para la pesca deportiva por sus característica combativas. El desarrollarse entre los 18 y $28^{\circ} \mathrm{C}$ la convierte en una especie de cultivo promisoria. Un estudio citogenético a partir de células branquiales indicó diferentes complementos cromosómicos. Este trabajo aplicó la técnica de cultivo de linfocitos a muestras de sangre de adultos. Las muestras de sangre total fueron sembradas en $4 \mathrm{ml}$ de medio de cultivo Ham-F12, suplementado con $0.5 \mathrm{ml}$ de Suero Fetal Bovino y $0.7 \mathrm{ml}$ del mitógeno Fitohemaglutinina $\mathrm{M}\left(\mathrm{Gibco}{ }^{\circledR}\right)$ durante $96 \mathrm{hr}$ a $28^{\circ} \mathrm{C} ; 1.5 \mathrm{hr}$ antes de la cosecha agregamos $100 \mu \mathrm{l}$ de Colcemid al $10 \%$ como factor antimitótico. Cada cultivo se incubó con $10 \mathrm{ml}$ de solución hipotónica $\mathrm{KCl} 0.56 \%$ por 24 min a $37^{\circ} \mathrm{C}$, para continuar con tres fijaciones sucesivas, centrifugaciones y tinción Giemsa al 5\%. Las mejores metafases fueron fotografiadas en microscopio, correspondientes a 6 machos y 8 hembras, indicando un número diploide de 50 cromosomas, clasificados en Metacéntricos (26M), Submetacéntricos (16SM) y Subtelocéntricos (8ST), para un número fundamental (NF) de 100 brazos. La tendencia evolutiva hallada fue de tipo B; no se encontraron cromosomas supernumerarios pero sí un primer par de cromosomas metacéntricos grandes, considerado marcador para el género Brycon, no 
determinante de heteromorfismo sexual. Estos resultados coinciden con los demás bricónidos investigados, en donde se podría considerar un ancestro común con un número cromosómico básico y cariotipos simétricos.

Palabras clave: Cariotipo, cromosomas, peces, bricónidos, linfocitos.

\section{REFERENCIAS}

Acuña, J.D. \& M.J. Bacca 2000. Caracterización de cromosomas en células del riñón de bocachico Prochilodus magdalenae Steindachner, 1878 (Pisces: Prochilodontidae) mediante la técnica de bandeo G. Tesis de Biólogo, Universidad del Atlántico, Barranquilla, Colombia.

Arias-Rodriguez, L.; Páramo-Delgadillo, S. \& A. de la Luz Durán-González. 2006. Caracterización citogenética del pez tropical de agua dulce Parachromis managuensis (Pisces: Cichlidae). Re. Biol. Trop. 54: $35-42$.

Arreguín- Espinosa, J. \& M. Uribe-Alcocer. 1989. Los cromosomas de los peces Oreochromis urolepis hornorum y Oreochromis mossambicus (Pisces: Cichlidae). Inst. Cienc. Mar y Limnol., UNAM, México. 16: 189-198.

Brassesco, M.S., M.C. Pastoril, H.A. Roncati \& A.S. Fenocchio. 2004. Comparative cytogenetic studies of Curimatidae (Pisces, Characiformes) from the middle Parana River (Argentina). Genet. Mol. Res. 3: 293-301.

Bertollo, L., C.S. Takahashi \& O. Moreira-Filho. 1978. Cytotaxonomic considerations on Hoplias lacerdae (Pisces, Erythrinidae). Rev. Brasil. Genet. 1: 103120.

Bertollo, L., G.G. Born, J.A. Dergam, A.S. Fenocchio \& O. Moreira-Filho. 2000. A biodiversity approach in the neotropical Erythrinidae fish, Hoplias malabaricus. Karyotypic survey, geographic distribution of cytotypes and cytotaxonomic considerations. Chromosome Res. 8: 603-613.

Born, G.G. \& L.A. Bertollo. 2001. Comparative cytogenetics among allopatric populations Hoplias malabaricus. Cytotypes with $2 \mathrm{n}=42$ chromosomes. Genetica 110: $1-9$.

Boron, A. 2003. Karyotypes and cytogenetic diversity of the genus Cobitis (Pisces, Cobitidae) in Poland: a review. Cytogenetic evidence for a hybrid origin of some Cobitis triploids. Folia Biol. (Krakow). 51: $49-54$.
Builes, J \& A. Urán. 1974. Estudio del ciclo sexual de la sabaleta Brycon henni Eigenmann. Su comportamiento y fecundidad artificial. Rev. Act. Biol. 3: 2-5.

Burbano, C. 2001. Citogenética aplicada a peces, p 219232. En: H. Rodríguez, P.V. Daza \&, M. Carrillo (eds). Fundamentos de Acuicultura Continental. Grafiimpresos Quintero. Bogotá, Colombia.

Camacho- Garzón, J. \& C. Burbano. 1999a. Caracterización citogenética de Pseudoplatystoma fasciatum (Bagre rayado) y Pseudoplatystoma tigrinum (Bagre tigre) (Pisces: Siluriformes: Pimelodidae). Tesis de Biólogo, Universidad Nacional de Colombia, Bogotá, Colombia.

Camacho-Garzón, J \& C. Burbano. 1999b Técnica para el cultivo in vitro de linfocitos de peces. Dahlia. 3: 69-79.

Carolsfeld, J., B. Harvey, C. Ross \& A. Baer. 2004. Migratory fishes of South America. Biology, Fisheries and Conservation Status. The International Bank for Reconstruction and Development / The World Bank., Ottawa, Canada.

Castillo, L. \& E. Rubio. 1986. Estudio de la ictiofauna de los esteros y partes bajas de los ríos San Juan, Dagua y Calima, Departamento del Valle del Cauca. Cespedesia. 15: 53-56.

Chen, T.R. \& A.W. Ebeling. 1971. Chromosomes of the goby fishes in the genus Gillchthus. Copeia. 1: 171-174.

Dahl, G. 1971. Los peces del Norte de Colombia. Inderena, Ministerio de Agricultura, Bogotá, Colombia.

De Almeida-Toledo, L.F., A.P. Bigoni, G. Bernadino \& S.A. Toledo-Filho. 1995. Chromosomal location of NORs and Bands in F1 hybrids of bighead carp and silver carp reared in Brasil. Aquaculture 135: 227-284.

De Greiff, S. \& F. Montoya. 1988. Estudio genético y bioquímico de cuatro especies del género Brycon de origen colombiano. Universidad Nacional de Colombia, Medellín, Colombia.

De Robertis, E.D \& E. Robertis. 1986. Biología celular y molecular. Ateneo, Buenos Aires Argentina.

Dos Santos, A.C. \& S. Morelli. 2000 Karyotypic study in population of Astyanax scabripinnis (PiscesCharacidae) from Jatai Stream headwater, UberlandiaMG. Hor. Cient. 1: 1-16.

Durán- González, A, C.E. García-Rucias \& A. LaguardaFigueras. 1990. The karyotype and " $\mathrm{G}$ " bands of Haemulon aurolineatum CLUVIER, 1829 (Pisces: 
Haemulidae). An. Inst. Cienc. del Mar y Limnol. Univ. Nal. Auton. México. 17: 299-307.

Durán- González, A \& A Laguarda. 1992. Caracterización citogenética del Marlín Blanco Tetrapturus albidus Poey, 1860 (Pisces: Istiophoridae) del Caribe mexicano. An. Inst. Cienc. del Mar y Limnol. 19: 143-150.

Fenocchio, A.S., L.A. Bertollo, C.S. Takahashi \& J.P.Camacho. 2000. B chromosomes in two fish species, Genus Rhamdia (Siluriformes, Pimelodidae). Folia Biol. 48:105-109.

Fenocchio, A.S. \& L.A. Bertollo. 1988. A simple method for fresh-water fish lymphocyte culture. Rev. Brasil. Genet. 11: 847-852.

Feldberg, E., J.I. Revelo, E.B. Pedraça \& F.C. Souza. 1999. Cytogenetic studies of two freshwater sciaenids of the genus Plagioscion (Perciformes, Sciaenidae) from the central amazon. Genet. Mol. Biol. 22: 351-356.

Fitzsimons, J.M. 1972. A revision of two genera of Goodeid fishes (Cyprinodontiformes, Osteichthyes) from the mexican plateau. Copeia. 4: 728-756.

Freshney, I. 2000. Culture of Animal Cells. A manual of basic technique. Willey Book, Nueva York, Nueva York, EEUU.

Howell, W.M. \& J. Villa. 1976. Chromosomal homogenety in two Sympatric cyprinid fishes of the genus Rhnichthys. Copeia 1: 112-116.

Instituto Alexander von Humboldt. 2000. Colombia mega diversa: cinco años explorando la riqueza de un país biodiverso. Instituto de Investigación de Recursos Biológicos Alexander von Humboldt. Villa de Leyva. Boyacá, Colombia.

Jankun, M. \& P. Ráb. 1997. Multiple polymorphism of chromosome no.1 in the karyotype of whitefish, Coregonus lavaretus (Salmonidae) from lake system Saimaa, Finland. Caryologia. 50: 185-195.

Kavalco, K.F., R. Pazza, L.A. Bertollo \& O. MoreiraFilho. 2005. Karyotypic diversity and evolution of Loricariidae (Pisces, Siluriformes). Heredity. 94: 180-186.

Kavalco, K.F., R. Pazza, L.A. Bertollo \& O. Moreira-Filho. 2005. Molecular cytogenetics of Oligosarcus hepsetus (Teleostei, Characiformes) from two Brazilian locations. Genetica. 124: 85-91.

Klinkhardt, M, M. Tesche \& H. Greeven H. 1995. Database of Fish Chromosomes Westarp-Wissenschaften, Magdeburg, Alemania.
Lehman, P. 1999. Composición y estructura de las comunidades de peces de dos tributarios en la parte alta del río Cauca, Colombia. Cespedesia. 23:73-74

Levan, A., K. Fredga, A.A. Sandberg, A. Gropp \& H Winking. 1964. Nomenclature for centromeric position of chromosomes. Hereditas. 52: 201-220.

Margarido V. \& P. Galetti. 1999. Heterochromatin patterns and karyotype relationships within an between the genera Brycon and Salminus (Pisces, characidae). Genet. Mol. Biol. 22: 357-361.

Martino, G. 2004. Avances en la obtención de cariotipos en las especies Morocoto Piaractus brachypomus, del híbrido F1 de Colossoma macropomum (hembra) por Piaractus brachypomus (macho) y el híbrido F2 retrocruzado de híbrido $\mathrm{F} 1$ hembra por macho de sus especies parental. Comunicación Científica CIVA 2004. 352-357 (also available on-line: www.revistaaquatic.com/civa2004/coms/completo.asp?cod=18).

Miles, C. 1947. Los peces del río Magdalena; El Gráfico, Bogotá, Colombia.

Montoya-López, A.F., L.M. Carrillo \& M. Olivera-Angel. 2006. Algunos aspectos biológicos y el manejo en cautiverio de la Sabaleta Brycon henni, Eigenmann, 1913 (Pisces: Characidae). Rev. Col. Cienc. Pec. 19: 180-186.

Molina, B. 1982. Reproducción inducida de la Sabaleta. Seminario presentado como requiso parcial para optar al título de Zootecnista, Universidad Nacional de Colombia. Medellín, Colombia.

Molina, W.F. \& Galetti P.M. 2002. Robertsonian rearrangements in the reef fish Chromis (Perciformes, Pomacentridae) involving chromosomes bearing $5 \mathrm{~s}$ rRNA genes. Genet. Mol. Biol. 25: 373-377.

Morgan, S.J. \& D.C. Darling. 1995. Cultivo de Células de animales. Acribia, Mdrid, España.

Nakayama, C.M. Jégu, J.I. Porto \& E. Feldberg. 2001. Karyological evidence for a cryptic species of piranha within Serrasalmus rhombeus (Characidae, Serrasalminae) in the Amazon. Copeia: 3: 866-869.

Nakayama, C.M., J.I. Rebelo \& E. Feldberg. 2002. A comparative cytogenetic study of five piranha species (Serrasalmus, Serrasalminae) from the Amazon basin. Genetica: 114: 231-236.

Paintner-Marques T.R., L. Giuliano-Caetano \& A.L. Dias. 2003. Cytogenetic characterization of a population of Bryconamericus aff. iheringii (Characidae, Tetragonopterinae). Genet. Mol. Biol. 26: 145-149. 
Parada, S., J.A. Arias \& P.E. Cruz. 2003. Caracterización cariotípica del yamú (Brycon siebenthalae). Rev. Orinoquia. 7:42-46

Pareja, D.A. 2002. Estudio de la variación genética de Brycon henni del departamento de Antioquia mediante RAPD-PCR. Tesis de Biólogo, Universidad de Antioquia, Medellín, Colombia.

Perdomo, J. 1978. La Sabaleta (Brycon henni) observaciones bioecológicas y su importancia como especie de cultivo. Rev. Div. Pesq. 11:1-46

Phillips, R.B., Konkol, N.R., Reed K.M. \& J.D. Stein. 2001. Chromosome painting supports lack of homology among sex chromosomes in Oncorhynchus, Salmo, and Salvelinus (Salmonidae). Genetica. 111: 119-123.

Pineda, H., L. Arboleda, A. Echeverry, S. Urcuqui, D. Pareja, M. Olivera \& J. Builes. 2007. Caracterización de la diversidad genética en el pez Brycon henni (Characiformes: Characidae) en Colombia central por medio de marcadores RAPD. Rev. Biol. Trop. 55: $1025-1035$.

Porto-Foresti, F.C., E. Oliveira, E. Gomes, Y. Tabata, M. Rigolino \& M.A. Foresti Marcos. 2004. A letal effect associated with polymorphism of the NOR- bearing chromosomes in rainbow trout (Oncorhynchus mykiss). Genet. Mol. Biol. 27: 51-54.

Saez, F. \& H. Cardoso. 1978. Citogenética básica y biología de los cromosomas. V. Chesneau, Washington, EEUU.

Saito, Y., Edpalina, R. \& S. Abe 2007. Isolation and characterization of salmonid telomeric and centromeric satellite DNA sequences. Genetica 131: 157-166.

Salas, E. \& J. Boza. 1991. Citotaxonomía comparativa de tres especies de Cichlasoma (Pisces: Cichidae) nativas de Costa Rica. Rev. Biol. Trop. 39: 219-224.

Sato, R.L., C. Oliveira \& F. Foresti. 2004. Karyotype description of five species of Trichomycterus (Teleostei: Siluriformes: Trichomyctaridae). Genet. Mol. Biol. 27: $45-50$.

Silva, A. 2001. Caracterización citogenética del bocachico Prochilodus reticulatus Valenciennes 1850 (Characiformes: Curimatedae) de una población colombiana del río Magdalena. Tesis de Biólogo, Universidad Nacional de Colombia, Bogotá, Colombia.

Sola, L., P.J. Monaco \& E.M. Rasch. 1990. Cytogenetics of bisexual/unisexual species of Poecilia. I. C-bands,
Ag-Nobn R polymorphisms, and sex chromosomes in three populations of Poecilia latipinna. Cytogenet. Cell. Genet. 53:148-54.

Swarça, A.C., A.S. Fenocchio, M.M. Cestari \& A.L. Dias. 2005. First chromosome data on Steindachneridion scripta (Pisces, Siluriformes), Pimelodidae from Brazilian rivers: Giemsa, CBG, G-, and RE banding. Genet. Mol. Res. 4: 734-741.

Therman, E. \& M. Susman. 1993. Human chromosomes: Structure, Behavior and Effects. Springer; Nueva York, EEUU.

Thompson, K. 1979. Cytotaxonomy of 41 species of neotropical cichlidae. Copeia 4: 679-691.

Tripathy, N.K. \& C.C. Das. 1988. Karyotypes of five Indian Perciform fishes. Copeia 1: 231-233.

Uribe-Alcocer, M., J. Arreguín-Espinosa, A. TorresPadilla \& A. Castro-Pérez. 1983. Los cromosomas de Dormitador latifrons (Pisces: Gobiidae). An. Inst. Cienc. del Mar y Limnol. Univ. Nal. Auton. México 10: 23-30.

Uyeno, T. \& R.R. Miller. 1973. Chromosomes and the evolution of the Plagopterin fishes (Cyprinidae) of the Colorado River System. Copeia. 4: 776-787.

Valenzuela, E. 2001. Caracterización citogenética del blanquillo Sorubim cuspicaudus (Siluriformes: Pimelodidae) de una población colombiana del río Sinú. Tesis de Biólogo, Universidad Nacional de Colombia, Bogotá, Colombia.

Vicente, V.E., O. Moreira-Filho \& J.P. Camacho. 1996. Sex-ratio distortion associated with the presence of a B chromosome in Astyanax scabripinnis (Teleostei, Characidae). Cytogenet. Cell. Genet. 74: 70-5.

Wasco, A. P. \& P.M. Galetti. 2000. Mapping 18S ribosomal genes in fish of the genus Brycon (Characidae) by fluorescence in situ hybridization (FISH). Genet. Mol. Biol. 23: 135-138.

Wosnicki, P, M. Jankun, D. Kucharczyk, A. Boron \& M. Luczynski. 1999. Cytogenetic characterization of Sea Trout (Salmo trutta) from Poland. Copeia: 2: 501-505.

Zapata, L. \& R. Vanegas. 1993. Aspectos importantes sobre la reproducción inducida y el metabolismo de las gónadas de la Sabaleta Brycon henni E. Tesis de Biólogo, Universidad de Antioquia, Medellín, Colombia. Trabajo de grado para obtener el título de Biólogos Medellín, Colombia. 